Rev SINAPSIS, Vol. 6, No 1, Junio 2015

ISSN $1390-7832$

\title{
Estrategia didáctica para la comprensión lectora en inglés con Fines Profesionales para estudiantes de Veterinaria y Zootecnia.
}

\section{Didáctica para la comprensión lectora en estudiantes Veterinarios.}

\author{
Maritza Núñez Arévalo (1) \\ Ana Velia Domínguez León ${ }^{(2)}$ \\ (1)Universidad de Matanzas, Matanzas, Cuba. \\ ${ }^{\text {(2)} U n i v e r s i d a d ~ d e ~ G r a n m a, ~ C u b a ~}$
}

\section{Contacto: maritza.nunez@umcc.cu}

Receptado: 22/03/2015 Aceptado: 11/05/2015

\section{Resumen}

El presente trabajo, muestra un recorrido por los momentos y tendencias principales sobre la impartición del idioma extranjero, se realiza un análisis de la situación actual a partir de la introducción del enfoque comunicativo que ha revolucionado la enseñanza del inglés en las universidades cubanas, con la introducción de la semipresencialidad y la Clase Encuentro (CE) como tipología de clase, Este artículo permite socializar una variedad de ejercicios creadores y motivadores que dé lugar a la continuidad del desarrollo de las cuatro habilidades lingüísticas (escuchar, hablar, leer y escribir), es por ello que tiene como objetivo presentar una propuesta de ejercicios a través de una estrategia didáctica para la comprensión lectora teniendo en cuenta los pasos para entender correctamente un texto; así como desarrollar habilidades creativas y motivadoras a través de los ejercicios retadores. Se concluyó que se contribuye a la formación integral del futuro profesional aportándole nuevas vías a través de la estrategia presentada para el tratamiento de los textos de lectura en el Inglés con Fines Profesionales, además de vincularlos directamente con el uso práctico y útil de la lengua Inglesa con un Enfoque Comunicativo teniendo en cuenta los momentos de la lectura.

Palabras clave: Adquisición y construcción del conocimiento, enfoque comunicativo, estrategia didáctica, tipologías de ejercicios

Didactic strategy for reading comprehension in English with professional purposes for Veterinary students 
Rev SINAPSIS, Vol. 6, No 1, Junio 2015

ISSN $1390-7832$

\section{Abstract}

The present research, reflects a course for the main moments and tendencies about the English teaching, in addition to, it is stopped in an analysis of nowadays situation of the introduction of the communicative approach that have transformed the English teaching in Cuban universities, with the introduction of semipresence lessons as exercise typology, it allows to socialize a variety of creative and native exercises that gives the possibility to continue the development of the four skills (listening, speaking, reading, and writing), that's why it has as objective to present a variety of exercises though a didactic strategy for the reading comprehension taking into account the steps to understand the text correctly; so to develop creative and motivated activities though impact exercises. As conclusion, it is contributed to the future professional integral formation giving new ways though the presented strategy for the work of the reading texts in the English of professional purposes, besides, connect them directly with the practical and useful use of English language with a communicative approach taking into account the moments of the reading.

Keywords: acquisition and construction of knowledge, communicative approach, typology of exercise

\section{Introducción}

En los últimos años, la política educacional ha estado orientada a formar ciudadanos con una cultura general integral y con un pensamiento humanista, científico y creador, que les permita adaptarse a los cambios de contexto y resolver problemas de interés social con una ética y una actitud crítica y responsable, a tono con las necesidades de una sociedad que lucha por desarrollar y mantener sus ideas y principios en medio de enormes dificultades y desafíos. También resulta necesario tener en cuenta la necesidad de comunicación entre especialistas de diversas nacionalidades en diferentes lenguas dado el incremento de las interrelaciones profesionales a nivel internacional (Breen, 1997; Cartón, 2002).

En el proceso de enseñanza aprendizaje de la lengua inglesa, el desarrollo de las habilidades lingüísticas, así como el de las destrezas intelectuales, requieren de mucha creatividad para el docente, pues son procesos muy complejos que tienen implicaciones psicológicas, los estudiantes de las universidades son jóvenes, por lo que el profesor debe propiciar la motivación para lograr dar respuestas a sus expectativas (Antich, 1978).

La necesidad de un acercamiento al texto ha provocado que especialistas, y profesores de todo el mundo busquen diferentes modos de actuación frente al problema de la comprensión 
Rev SINAPSIS, Vol. 6, No 1, Junio 2015

ISSN $1390-7832$

textual. No debe ser una sorpresa tal empeño, si se tiene en cuenta que la lectura, por sus propias características - situación interlocutiva indirecta- "desplaza al emisor y exige una mayor actividad por parte del lector" En efecto, este se ve obligado a tomar decisiones, a elegir y a reconstruir el texto. En este mismo sentido, afirma con razón Paulo Freire: "La comprensión de un texto no es algo que se recibe de regalo: exige trabajo paciente de quien se siente problematizado por él" (Otero 2011) aborda el tema refiriéndose a la preparación que deben tener los docentes de Inglés para desarrollar una adecuada comprensión lectora.

Por lo que se propuso aplicar una estrategia didáctica para mejorar la comprensión de textos en el inglés con fines profesionales a través de ejercicios creativos y motivadores que estimulen el esfuerzo de los estudiantes, así como será de gran utilidad para la preparación metodológica de los profesores en la modalidad semipresencial, teniendo en cuenta los niveles de lectura.

La estrategia didáctica para la comprensión de textos se plantea como objetivo general: ofrecer las bases teóricas didácticas indispensables para el proceso de enseñanza aprendizaje de la competencia comunicativa, de manera que se influya progresivamente en el desarrollo de la comprensión de textos para los estudiantes de veterinaria en el inglés con fines específicos; donde se toma como específico: instrumentar y evaluar en la práctica escolar un sistema de acciones docentes que faciliten el perfeccionamiento de la dirección del aprendizaje para la comprensión de texto.

\section{Materiales y métodos}

Para la puesta en práctica de la estrategia se utilizó diferentes fuentes bibliográficas, las cuales sirvieron como base teórica para el tratamiento y solución del problema. Se realizó un diagnóstico a 301 estudiantes, la cual que dio la posibilidad del conocimiento para observar el modo de actuación, es decir, para saber sobre el comportamiento de los estudiantes que se tomaron como muestra. En sentido, dicho diagnóstico se tomó como uno de los primeros pasos para un proceso de planeación estratégica llevado a cabo en el campo investigativo.

Para el análisis estadístico se empleó el software Statistcs v.8, lo que demostró la factibilidad de aplicación de la propuesta y por consiguiente el aumento de los niveles de dominio de la habilidad de lectura en los estudiantes que participaron en la intervención. 
Rev SINAPSIS, Vol. 6, No 1, Junio 2015

ISSN $1390-7832$

\section{Resultados}

Fundamentación de la estrategia didáctica para la comprensión de textos:

Desde el punto de vista filosófico, la estrategia asume que el desarrollo del lenguaje y el del celebro están directamente relacionadas, en la transformación del hombre desde un homínido hasta la especie humana actual, destacando las relaciones sociales, como un factor determinante en esta evolución a través del trabajo.

Desde lo psicológico, el enfoque Socio- Histórico Cultural de Vygotsky, en lo correspondiente a la Zona de Desarrollo Próximo. Se asume además la unidad de lo cognitivo y lo afectivo en el proceso de formación de la personalidad y del dominio a desarrollar (Vygotsky, 1973).

Para ello es necesario que este domine el sistema de conocimientos que le permitirá dar solución a la misma y con esto logre una adecuada presencia de sentimientos, necesidades, aspiraciones y motivos que le permitan una actuación correcta de su personalidad. Además de enriquecerlos continuamente a través de un proceso de comunicación afectivo. En este caso lo social y lo individual convergen y el estudiante se motiva por la tarea, sintiendo la necesidad de aprender. Se hace necesario tener en cuenta el estado actual del aprendizaje del objeto de estudio y potenciarlo durante la interacción social entre estos y el profesor o compañeros de grupo. Todos los métodos en su momento han sido totalmente reemplazados por otros, que a su vez han sido sustituidos por otros más modernos. "De igual forma los ya vigentes evolucionarán, serán modificados o reemplazados por otros (Ruiz, 2003).

Desde lo pedagógico, la enseñanza del idioma inglés en las universidades persigue como aspiración contribuir a la ampliación de la formación político ideológico, científico técnica y estética mediante la adquisición de los conocimientos y el desarrollo de las habilidades y hábitos necesarios para el aprendizaje de una lengua extranjera.

Desde la didáctica, el enfoque comunicativo, de Rodolfo Acosta Padrón, al considerar que para lograr ser un comunicador competente no solo se requiere desarrollar la competencia comunicativa, sino una competencia comunicativa intercultural y educativa. De Montano, la concepción de las actividades previas a la lectura, durante la lectura y posterior a la lectura (Montano, 2005). 
La estrategia como resultado científico es considerada como: "la proyección de un sistema de acciones a corto, mediano y largo plazo que permite la transformación del proceso de enseñanza aprendizaje en una asignatura, nivel o institución tomando como base los componentes del mismo y que permite el logro de los objetivos propuestos en un tiempo concreto".

En el contexto de la didáctica, las estrategias sirven para planificar, tomar decisiones, controlar la aplicación de los métodos, procedimientos didácticos y poder adaptarlos a las necesidades específicas de cada situación docente (Álvarez, 1999). La misma requiere de premisas para su aplicación; ellas, actúan fuera del proceso e independientemente de la voluntad del que ejecuta la estrategia (Rodríguez, 2001).

Se establecieron cinco etapas:

Primera etapa: diagnóstico de la comprensión de texto

Segunda etapa: planificación de la comprensión de texto

Tercera etapa: ejecución de la comprensión de textos

Cuarta etapa: control de la comprensión de textos

Quinta etapa: retroalimentación de la comprensión de textos

Se proyectó un sistema de actividades que se basó en diferentes aspectos metodológicos:

Sugerencias metodológicas generales

De manera general, para todos los sistemas de actividades, es necesario que el maestro en el trabajo con el vocabulario tenga en cuenta su metodología; primero por el contexto, aprovechándose de aciertos y desaciertos, después con ayuda del grupo, y por último, buscando la palabra en el diccionario.

Se pudo establecer, además que el maestro puede crear nuevos sistemas de actividades, teniendo en cuenta las particularidades de los estudiantes, así como los componentes que tengan dañados, haciéndolo desde la comprensión textual, utilizando en el aula, trabajo por parejas o pequeños grupos.

Estos sistemas de actividades deben utilizarse en las clases de comprensión de textos, los mismos servirán para desarrollar el dominio cognitivo objeto de estudio. 
Rev SINAPSIS, Vol. 6, Nº 1, Junio 2015

Se le recomienda al profesor, para aprovechar el tiempo al máximo, llevar los textos copiados con anterioridad para repartirlos por parejas o pequeños grupos, o que estos los tengan con anterioridad.

Cada sistema de actividades se corresponde con las asignaturas que reciben en segundo año y cada una tiene sugerencia metodológica particular.

El docente debe tener en cuenta los tres momentos de la lectura y de esta misma forma preparar actividades teniendo en cuenta los diferentes niveles de adquisición.

Se corroboró que la lectura debe estar centrada en tres momentos:

- Actividades de anticipación o preparación para la lectura.

- Actividades durante la lectura

- Actividades después de la lectura

Esta propuesta se aplicó en el grupo 301 de la carrera de Veterinaria y zootecnia de la Universidad de Granma, el cual se le aplicó un diagnóstico inicial que consistió en una evaluación escrita la cual corroboró las insuficiencias existentes en los estudiantes dadas en problemas al seleccionar ideas generales, principales y secundarias de los textos, buscar sinónimos, antónimos, contestar preguntas, realizar outline, hacer resúmenes, entre otras. Esto demostró la necesidad de utilizar la Estrategia propuesta para contribuir al perfeccionamiento del proceso de enseñanza aprendizaje de la lectura.

Una vez culminada la aplicación práctica de la misma, se realizó a los estudiantes una prueba de salida que demostró la pertinencia y validez de la propuesta. En este sentido se compararon los resultados del diagnóstico inicial con los de la prueba de salida a través de la utilización de técnicas estadísticas interpretadas. Se comenzó el análisis estadístico descriptivo determinando los estadígrafos de posición y dispersión (Tabla 1).

Tabla 1. Estadística descriptiva.

\begin{tabular}{|c|c|c|c|c|c|c|c|c|c|c|c|}
\hline & $\begin{array}{l}\text { Valid } \\
\text { N }\end{array}$ & Mean & $\begin{array}{c}\text { Media } \\
\mathbf{n}\end{array}$ & Mode & $\begin{array}{c}\text { Frequenc } \\
\text { y - of } \\
\text { Mode }\end{array}$ & $\begin{array}{l}\text { Mini } \\
\text { m. }\end{array}$ & $\begin{array}{c}\text { Maxi } \\
\text { m. }\end{array}$ & $\begin{array}{c}\text { Varia } \\
\text { n }\end{array}$ & $\begin{array}{c}\text { Std.De } \\
v\end{array}$ & $\begin{array}{c}\text { Coef.Va } \\
\text { r. }\end{array}$ & $\begin{array}{c}\text { Standar } \\
\mathrm{d} \text { - } \\
\text { Error }\end{array}$ \\
\hline $\begin{array}{c}\text { Notas } \\
\text { diagnóstico }\end{array}$ & 23 & $\begin{array}{l}2.826 \\
087\end{array}$ & 3.0000 & $\begin{array}{l}3.0000 \\
0\end{array}$ & 12 & $\begin{array}{l}2.000 \\
0\end{array}$ & $\begin{array}{l}5.000 \\
0\end{array}$ & $\begin{array}{l}0.604 \\
7\end{array}$ & \begin{tabular}{|l|}
0.7776 \\
5
\end{tabular} & 27.5169 & $\begin{array}{l}0.16215 \\
2\end{array}$ \\
\hline Notas & 23 & 3.869 & 4.0000 & 4.0000 & 10 & 3.000 & 5.000 & 0.573 & 0.7570 & 19.5641 & 0.15785 \\
\hline
\end{tabular}


Rev SINAPSIS, Vol. 6, No 1, Junio 2015

\begin{tabular}{|c|l|l|l|l|l|l|l|l|l|l|}
\hline $\begin{array}{c}\text { Evaluación } \\
\text { final }\end{array}$ & 565 & 0 & & 0 & 0 & 1 & 4 & & 6 \\
\hline
\end{tabular}

\begin{tabular}{|c|l|l|l|l|l|}
\hline \multicolumn{1}{|c|}{} & Count & Cumulative - Count & Percent & Cumulative - Percent \\
\hline 3 & 8 & 8 & 26.66667 & 26.6667 \\
\hline 4 & 10 & 18 & 33.33333 & 60.0000 \\
\hline 5 & 5 & 23 & 16.66667 & 76.6667 \\
\hline Missing & 7 & 30 & 23.33333 & 100.0000 \\
\hline
\end{tabular}

Como se puede observar, los valores de la media, la mediana y la moda son superiores en la

aplicación de la estrategia en la evaluación final con relación al diagnóstico, siendo la nota de 3 puntos en el diagnóstico la puntuación que más se repite (12 veces) y en la evaluación final, la nota que más frecuencia tiene es de 4 puntos, la cual se repite 10 veces.

Asimismo, con la aplicación del diagnóstico la nota mínima alcanzada por los estudiantes fue de 2 puntos y en la evaluación final de 3. En lo que respecta a la nota máxima, en el diagnóstico fue de 5 puntos al igual que en la evaluación final.

Por su parte, la determinación de la varianza demostró que el diagnóstico, al tener valores superiores con respecto a la evaluación final, tiene mayor dispersión.

La desviación típica en el diagnóstico nos indica que las calificaciones se desvían como promedio en 0.77 puntos de su media (2.82 puntos, 2 puntos redondeado) y la desviación típica de la evaluación final demuestra que las calificaciones se desvían como promedio en 0.75 puntos de su media (3.86).

De igual forma, el coeficiente de variación en la evaluación final demuestra que hay menos dispersión, es decir, los datos están más concentrados.

Las medidas de dispersión indican que las 2 series de datos se encuentran concentradas cerca de sus respectivas medias aritméticas.

Además, se tabularon las frecuencias correspondientes para cada momento (Tablas 2 y 3 ).

Tabla 2. Tabla de frecuencia: notas evaluación final. 
Rev SINAPSIS, Vol. 6, No 1, Junio 2015

Tabla 3. Tabla de frecuencia: notas del diagnóstico.

\begin{tabular}{|c|l|l|l|l|l|l|}
\hline \multicolumn{1}{|c|}{} & Count & Cumulative - Count & Percent & Cumulative - Percent \\
\hline $\mathbf{2}$ & 8 & 8 & 26.66667 & 26.6667 \\
\hline $\mathbf{3}$ & 12 & 20 & 40.00000 & 66.6667 \\
\hline $\mathbf{4}$ & 2 & 22 & 6.66667 & 73.3333 \\
\hline $\mathbf{5}$ & 1 & 23 & 3.33333 & 76.6667 \\
\hline Missing & 7 & 30 & 23.33333 & 100.0000 \\
\hline
\end{tabular}

Interpretando las frecuencias absolutas tenemos que el $26.6 \%$ de los estudiantes con la aplicación del diagnóstico obtuvieron una calificación de 2 puntos y que el $3 \%$ alcanzó una calificación de 5 puntos. En la evaluación final tenemos que el 26.6\% de los estudiantes obtuvieron una calificación de 3 puntos y que el $16.6 \%$ alcanzó una calificación máxima de 5 puntos.

De forma general, los datos procesados mostraron que en el diagnóstico la puntuación de los estudiantes se encuentra con mayor frecuencia entre 2 y 3 puntos, mientras que en la evaluación final oscilan entre 3 y 5 puntos, con mayor frecuencia de 4 puntos, representados gráficamente en los histogramas de variables múltiples siguientes (Gráfico 1):

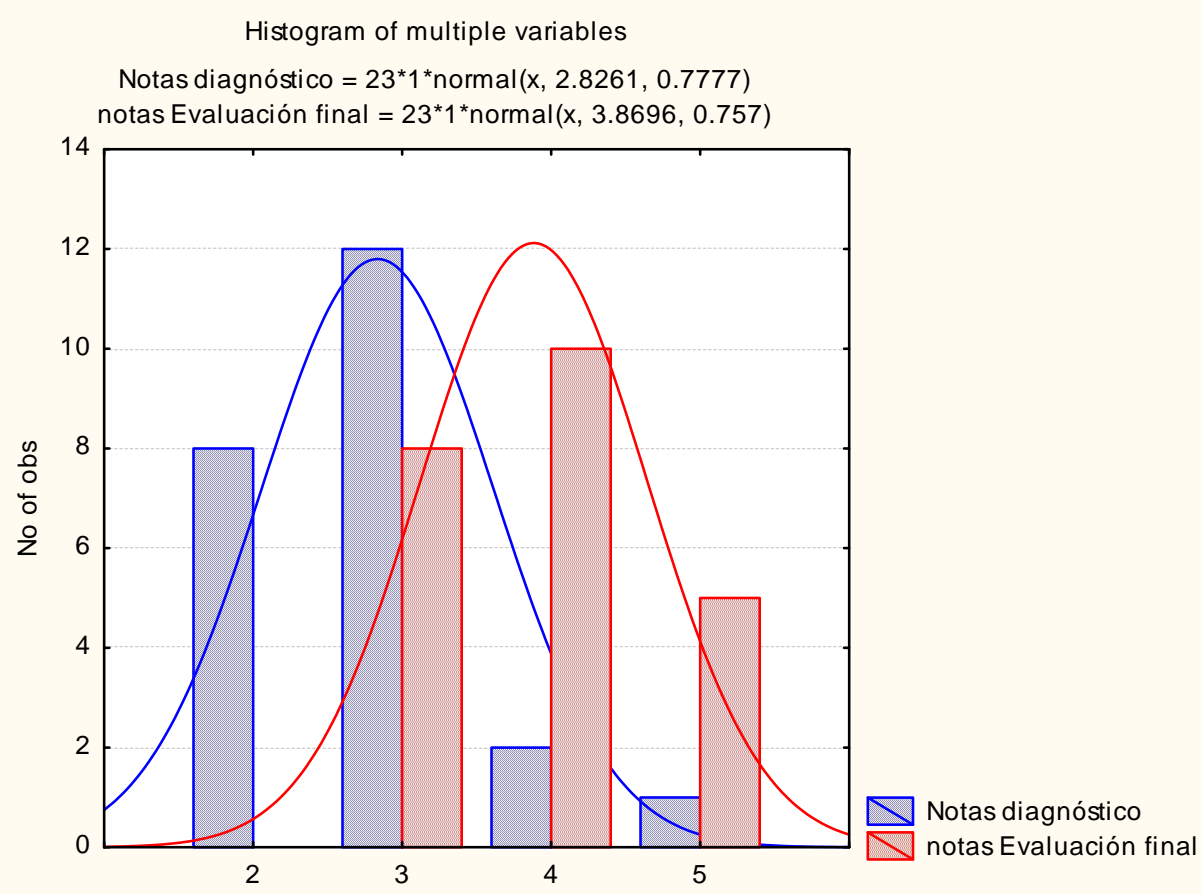

\section{Gráfico 1. Comparación entre las notas del diagnóstico y la evaluación final.}

Se realizó, además, la prueba de hipótesis de diferencia de medias para determinar la significatividad en cada caso (Tabla 4).

Tabla 4. Comparación entre las notas del diagnóstico y la evaluación final. 


\begin{tabular}{|c|c|c|c|c|c|c|c|c|c|c|}
\hline $\begin{array}{c}\text { Mean } \\
\text { Diagnóst } \\
\text { ico }\end{array}$ & \begin{tabular}{|c|} 
Mean \\
Evaluaci \\
ón final \\
-
\end{tabular} & $\begin{array}{c}\text { t- } \\
\text { value }\end{array}$ & df & p & \begin{tabular}{|c} 
Valid \\
$\mathbf{N}$ \\
Diagn \\
.
\end{tabular} & $\begin{array}{l}\text { Valid } \\
\mathbf{N} \\
\text { Eval. } \\
\text { final }\end{array}$ & $\begin{array}{c}\text { Std.Dev. } \\
\text { Diagnósti } \\
\text { co }\end{array}$ & $\begin{array}{c}\text { Std.Dev } \\
\text {. Eval. } \\
\text { final }\end{array}$ & \begin{tabular}{|c|} 
F- \\
ratio - \\
Varia \\
nza
\end{tabular} & $\begin{array}{c}\text { p - } \\
\text { Varian } \\
\text { za }\end{array}$ \\
\hline 2.826087 & 3.869565 & $\begin{array}{l}- \\
4.6110 \\
5\end{array}$ & 44 & $\begin{array}{l}0.0000 \\
34\end{array}$ & 23 & 23 & 0.777652 & $\begin{array}{l}0.75704 \\
9\end{array}$ & $\begin{array}{l}1.055 \\
17\end{array}$ & $\begin{array}{l}0.90090 \\
6\end{array}$ \\
\hline
\end{tabular}

Los resultados de la tabla anterior indican que no se observan discrepancias significativas en la dispersión, se puede observar que las desviaciones típicas son casi iguales ocurriendo lo mismo con el coeficiente de variación. Teniendo en cuenta los datos mostrados, se puede concluir que la diferencia es altamente significativa ya que $\mathrm{p} \approx 0.00$ es muy inferior a 0.05 ; por lo que, con la aplicación de la estrategia propuesta los estudiantes obtuvieron mejores resultados docentes que con el procedimiento tradicional evaluado a través del diagnóstico.

Valoración de la efectividad de la estrategia propuesta.

La comprensión de textos es un acto complejo, en cuyo proceso se integra, los saberes del lector. En dicho proceso el individuo va extrayendo sus propias conclusiones, verifica si son acertadas y hace correcciones cuando descubre sus errores. En resumen se convierte en un intérprete creador del texto. La misma ocupa un lugar significativo en el quehacer de los docentes y las universidades necesitan que este propósito sea alcanzado, por parte de los estudiantes, en apoyo de su aprendizaje, para lograr la competencia comunicativa y el perfeccionamiento del modo de actuación del profesor.

Descripción del proceso de implementación de la estrategia didáctica para la comprensión de textos en el departamento de idiomas.

Se aplica un sistema de actividades con requerimientos establecidos, lo que resulta válido para el desarrollo de la comprensión textual. En un inicio es preciso ofrecer niveles de ayuda a los estudiantes; pero en la medida que estas acciones son sistemáticas, los resultados logran ser mejores, viabilizando más independencia y actividad creadora. También es aconsejable aplicarlo a los materiales que se utilizan durante el año académico (Robinson, 1991.)

Como resultado de la implementación de la estrategia y la aplicación de la prueba pedagógica final, se hace palpable que los estudiantes logran mayores niveles de independencia en el desarrollo de la comprensión de textos, logrando ser un comunicador competente, siendo capaz de comprender lo que otros tratan de significar, de manera creadora favoreciendo su independencia. 
Rev SINAPSIS, Vol. 6, No 1, Junio 2015

ISSN $1390-7832$

La valoración de la estrategia didáctica para la comprensión de textos proporciona su concreción en el proceso de enseñanza aprendizaje de este dominio cognitivo, con apoyo de los restantes y con el sustento del enfoque comunicativo de Rodolfo Acosta Padrón y discurriendo con el antes, el durante y el después de leer de Montano.

\section{Discusión}

"La comprensión de textos en una lengua extranjera es un proceso complejo que permite al estudiante construir un paso fundamental hacia la adquisición de las demás competencias básicas" (Corpas, 2014). Es importante la atención de este aspecto en la enseñanza del idioma inglés en la preparación del futuro veterinario

Si se toma en cuenta las aserciones de Pinza y Astudillo (2014) sobre el tema de que la enseñanza de Inglés con objetivos específicos ayuda el aumento del vocabulario científico técnico de los estudiantes, así como, al desarrollo de hábitos de lectura. En la investigación queda sustentada que en los cursos de inglés con propósitos específicos se debe trabajar básicamente la lectura para búsqueda de información especializada. Esta práctica aumenta el aprendizaje del inglés, lo que a la vez promueve el trabajo en equipo y sobre todo proporciona mejoras la expresión y habilidad oral.

Se comparte también, las afirmaciones de Murcia (2000), Raimes, A. (1983) y Colomer (1997) quienes coinciden en que el acto de leer es un acto interpretativo que guía hacia un conjunto de razonamientos y hacia la construcción de una interpretación de un mensaje escrito a partir de la información que proporciona el texto. Se ha aprobado de la importancia de dicha aserción en la formación de los veterinarios y Zootecnistas a través del desarrollo de la habilidad lectora en inglés con propósitos profesionales.

Este trabajo como parte de una investigación llevada a cabo con estudiantes de Medicina Veterinaria sienta pautas para que muchos estudiosos del tema continúen en la búsqueda de nuevos métodos que se aplique a esta esfera de la enseñanza ya que se ha probado que la lectura en lenguas extranjeras tiene también gran importancia social, educativa y práctica.

\section{Conclusiones}

A través de toda la investigación se concluyó que se contribuye a la formación integral del futuro profesional aportándole nuevas vías a través de la estrategia presentada para el tratamiento de los textos de lectura en el Inglés con Fines Profesionales en la carrera de 
Rev SINAPSIS, Vol. 6, No 1, Junio 2015

ISSN $1390-7832$

medicina Veterinaria y Zootecnia, la cual les permite vincularse directamente con el uso práctico y útil de la lengua Inglesa con un Enfoque Comunicativo teniendo en cuenta los momentos de la lectura, y además se puede llevar a cabo en las diferentes carreras donde se imparta el inglés con fines específicos.

\section{Bibliografía}

1. Álvarez Álvarez, Luís. (1999) La lectura: pasividad o dinamismo”, en Pedagogía: Ministerio de Educación”. P13

2. Antich, R. (1986). Metodología de la Enseñanza de Lenguas Extranjeras. Cuba: Editorial Pueblo y Educación. Pag 23.

3. Breen, M. (1997). Paradigmas contemporáneos en la enseñanzade la lengua extranjera II. Revista Signos. Austria, (20): 58-65, Abril-Junio.

4. Carton E. (2002). The demand for higher education in The Netherlands, 1950-1999. Economics of Education Review. Vol. 24(6) ,651-663, December.

5. Colomer, T. (1997). Signos. La enseñanza de la comprensión lectora, 20, 10-15.

6. Corpas, MD. (2014) Análisis y evaluación de la comprensión lectora en inglés como lengua extranjera en educación secundaria obligatoria. Recuperado el 25 de marzo de 20013 en http://www.ual.es/revistas/PhilUr/pdf/PhilUr11.1.CorpasArellano.pdf

7. Montano Calcines, Juan Ramón. (2005). La enseñanza de la lectura y la comprensión de textos en la escuela. 9 p.

8. Murcia, Alice. (2000). Discourse and context in Language teaching. Cambridge University Press.

9. Otero, J. A.(2011). La preparación metodológica para la comprensión lectora de los docentes en formación de cuarto año de la carrera de Lenguas extranjeras desde la microuniversidad, http://www/eumed.net/libros/2011a/932/index.htm

10. Pinza, A y Astudillo A. (2013). La importancia de la lectura en el idioma inglés aplicando técnicas y lecturas motivadoras Recuperado el 25 de abril de 2015 en http://www.uteg.edu.ec/_documentos/file/pdf/Alejandro\%20Pinza\%20\%20Astudillo.pdf

11. Raimes, Ann. (1983). Techniques in teaching writing. New York: Oxford University Press

12. Robinson P. (1991). ESP Today: A Practitioner's Guide. Prentice Hall International. United Kingdom. 98

13. Rodríguez Palacios Alvarina. (2001). citado en La estrategia como resultado científico de la investigación educativa p 26

14. Ruiz, M. (2003) Aplicaciones Pedagógicas del informe empresarial en el aula del Inglés de los negocios: Implementación de recursos diversos escritos en contextos de especialidad. Tesis presentada en opción al grado científico de Doctor en Ciencias. España. 445 p.

15. Vigostky. L (1973). Historia de las funciones psíquicas superiores, La Habana, citado por Tabloide Maestría Romeu escobar, $57 \mathrm{p}$ 